\title{
Pelvic Angiosarcoma Around Total Hip Prosthesis
}

\author{
Alberto Sánchez-García, Alberto Pérez García and Enrique Salmerón González \\ Department of Plastic and Reconstructive Surgery, University Hospital La Fe, Valencia, Spain
}

\begin{abstract}
Angiosarcomas constitute less than $1 \%$ of primary bone malignancies. Their association with prosthetic material has been described, while their etiology remains unclear. We present a case of a 64-year woman with severe bleeding and hip joint osteolysis after an accidental fall. She had undergone prosthetic total hip arthroplasty many years back. The rapid recurrence of pelvic hematoma and the refractory infection of pelvic cavity made necessary to disarticulate the prosthetic hip joint, after several attempts to apply conservative treatment. Despite supportive treatment, she died. Autopsy revealed evidently dissemanted angiosarcoma of pelvic bones. Angiosarcoma of the bone should be considered in the differential diagnosis of patients with uncontrollable bleeding and osteolysis, due to the increase of life expectancy when prompt and combined treatment is initiated.
\end{abstract}

Key Words: Hemangiosarcoma, hip prosthesis, bone fractures.

How to cite this article: Sánchez-García A, García AP, González ES. Pelvic angiosarcoma around total hip prosthesis. J Coll Physicians Surg Pak 2019; 29 (Supplement 2):S132-S134.

\section{INTRODUCTION}

Angiosarcomas are infrequent, malignant, mesenchymal tumors which constitute less than $1 \%$ of primary bone malignancies. They are composed of anastomosing vascular channels lined by malignant endothelial cells. 1 Their etiology remains unclear, but they have been associated, among other factors, with vascular and orthopedic devices, such as hip joint prostheses. ${ }^{2}$ Early diagnosis and biopsy are fundamental for improved survival. Definitive diagnosis is achieved through histological and histochemical tests. However, due to their low incidence and scarce treatment alternatives available, prognosis is dismal. ${ }^{3}$ Herein, we present a case of a 64-year woman diagnosed with primary angiosarcoma of pelvic bones.

\section{CASE REPORT}

A 64-year woman was referred to our hospital from another healthcare centre. Two weeks prior, after an accidental fall, the patient had developed evident swelling around left hip and anterolateral aspect of the thigh, associated with functional impairment. The patient had undergone a left total hip arthroplasty (CharnleyKerboull prosthesis with polyethylene socket) in 1982, due to previous history of developmental hip dysplasia. The initial CT scan revealed a periprosthetic fracture,

Correspondence to: Dr. Alberto Sánchez-García, Department of Plastic and Reconstructive Surgery, University Hospital La Fe, Valencia, Spain

E-mail: asgarcia1992@hotmail.com

Received: November 05, 2018; Revised: March 31, 2019; Accepted: April 20, 2019 massive osteolysis on left hemipelvis, and unplanted Charnley hip prosthesis. The iliopubic and ischiopubic rami and the innominate line were also absent. The area was surrounded by an extensive hematoma which required urgent drainage in order to relieve the pain and swelling (Figure 1).

However, during the next two weeks after initial evaluation in the Emergency Department, the patient progressively became anemic due to hemorrhage, so an embolisation of branch of the left superficial femoral artery was performed, with no clinical or radiologic improvement. At that, the patient was transferred to our hospital. Throughout the hospitalisation stay, the patient continued to progressively become more anemic, without signs of acute bleeding, requiring repeated blood transfusions. The possibility of coagulation disfunction was ruled out by performing a complete hemostasis study.

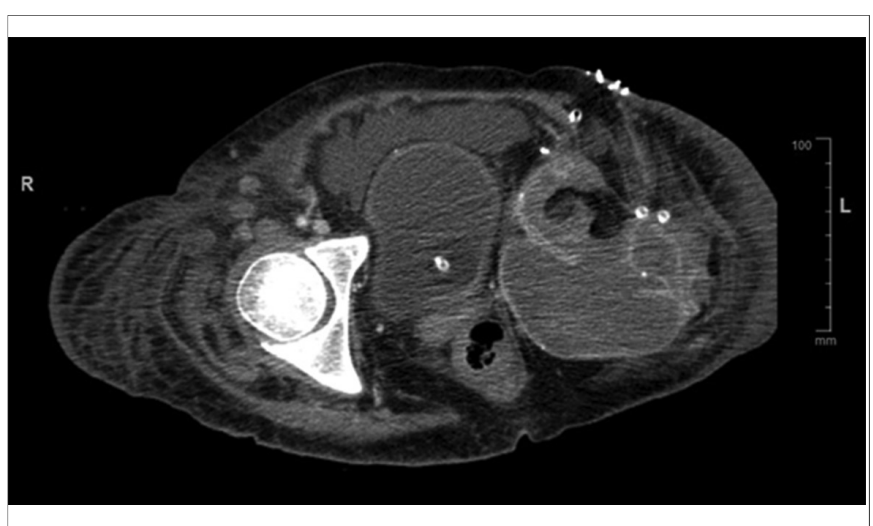

Figure 1: Digitally processed image of computerised tomography (CT) showing large collection located in left hemipelvis, as well as extensive bone destruction of the iliac blade and lateral segment of the left ischiopubic branch. 
A magnetic resonance imaging (MRI) scan showed a $34 \times 17 \times 17 \mathrm{~cm}$ lobulated and septated intra- and extrapelvic hematoma that reached proximal third of the left femur, affecting all thigh compartments, in contact with sciatic nerve. Hematoma evacuation was performed through a double ilioinguinal and anterolateral thigh approach, without detecting signs of acute bleeding. However, there was a rapid recurrence of the hematoma. During following weeks, hematocrit and hemoglobin decreased gradually. In addition to the drop in hematocrit, purulent material exited the surgical wound, and clinical signs of infection were observed.

In addition, a thoracic CT scan showed bilateral, partially loculated pleural effusions, which were larger on the left hemithorax. Left pleural drainage was performed, obtaining hemorrhagic content. Cytological examination gave negative result for malignant cells.

Finally, two weeks later, left hemipelvectomy and discharge colostomy were performed. After this intervention, the patient developed a global respiratory failure, which required orotracheal intubation. During the stay in the Critical Care Unit, the patient developed septic shock. High doses of norepinephrine were required. Finally, the patient presented a multi-organ failure, resulting in death.

Clinical autopsy was requested, which showed nodular accumulations of atypical cells in both lungs, pleura, diaphragm, left ventricle, right adrenal gland and bone marrow. The histological study of the excised hemipelvis showed occupation of the joint space by hemorrhagic material, as well as proliferation of atypical mesenchymal cells arranged in small cords. Those cells formed sinuous vascular channels and aggregated in cohesive groups within the bone, causing severe osteolysis. The immunohistochemical analysis tested positive for CD31 and ERG. The histopathological diagnosis of angio-sarcoma of the bone was then made.

\section{DISCUSSION}

Primary angiosarcoma of the bone is highly infrequent. Higher prevalence is seen in middle and older age groups, with a male:female ratio of 2:1. Angiosarcoma of the bone has a tendency to affect long bones like femur, tibia and fibula. ${ }^{4}$ Multifocal involvement of angiosarcoma of the bone is common, and it may result in difficulties in establishing the primary origin of the tumor, as in the case described; due also to the rapid locoregional dissemination, it usually presents.

Although several cases of association between prosthetic material and development of neoplasms have been described, there is not enough evidence to establish a cause and effect relationship between both. In adition, association between malignant neoplasms and hip arthroplasty deserves attention because this surgical procedure is so common that possible risk of neoplasm should be quantified. ${ }^{5}$

Early diagnosis with biopsy may be the only chance of the patient to survive. Definitive diagnosis is done with histological and immunohistochemical tests. The key of the immunohistochemical examination is the application of vascular markers for diagnostic studies. The most sensitive vascular marker is CD313.

Regarding treatment options, the approach must be individualised according to the degree of dissemination of the tumor and patient characteristics, being possible to opt for amputations or total or partial resections; always with adjuvant radiotherapy and/or chemotherapy.

According to Shen et al., in their review of 63 patients with angiosarcoma, while survival remains poor overall and recurrences rates high, combined therapeutic approach that includes surgical resection with taxanebased chemotherapy and/or radiotherapy improves survival compared to surgical resection alone. 6 In the case described, the diagnostic delay, coupled with the poor general condition presented by the patient during admission, made it impossible to follow an intensive combined treatment strategy.

Primary angiosarcoma of the bone is highly infrequent. Even so, it should be considered in the differential diagnosis of patients with uncontrollable bleeding and osteolysis, due to the increase of life expectancy when prompt and combined treatment is iniciated. Multifocal involvement of angiosarcoma of the bone is common; and it may result in difficulties in establishing the primary origin of the tumor, as in the case described, due also to the rapid locoregional dissemination it usually presents.

\section{PATIENTS' CONSENT:}

Informed consent was obtained from the patient included in this report.

\section{CONFLICT OF INTEREST:}

The authors declared that there is no potential conflict of interest relevant to this article.

\section{AUTHORS' CONTRIBUTION:}

ASG: Wrote the manuscript and collaborated in procedures. APG: Edited the manuscript and performed procedures. ESG: Edited the manuscript, obtained images, collaborated in procedures.

\section{REFERENCES}

1. Baliaka A, Balis G, Michalopoulou-Manoloutsiou E, Papanikolaou A, Nikolaidou A. Primary angiosarcoma of bone. A case report. Hippokratia 2013; 17:180-2.

2. Zhu W, Feng B, Ma Q, Li YL, Song XT, Weng XS. Angiosarcoma around hip joint prosthesis. Chin Med J (Engl) 2016; 129:2642-3. 
3. Oc Y, Kilinc BE, Ertugrul R, Sezer HB, Eren OT. Epithelioid angiosarcoma in femur: A case presentation. World $\mathrm{J}$ Oncol 2017; 8:196-8.

4. Akhavan Tafti M, Jafari N, Zare J, Jalal Jafari M. A case of bone angiosarcoma. Iran J Pathol 2016; 11:465-8.

5. Mallick A, Jain S, Proctor A, Pandey R. Angiosarcoma around a revision total hip arthroplasty and review of literature. J Arthroplasty 2009; 24:323.e17-20.

6. Shen CJ, Parzuchowski AS, Kummerlowe MN, Morris CD, Meyer CF, Habibi $\mathrm{M}$, et al. Combined modality therapy improves overall survival for angiosarcoma. Acta Oncol 2017; 56:1235-8.

......... 